\title{
One Management Scheme for Software Testing Based on TestCenter
}

\author{
Yue-Hua Ding \\ School of Mathematics and Computer Science, \\ Wuhan Polytechnic University, \\ Wuhan, China \\ E-mail: ding_mickey@126.com
}

\author{
Ri-Hua Xiang \\ Beijing Special Engineering Design and Research Institute, \\ Beijing, China \\ E-mail: xiangrihua@yahoo.com.cn
}

\begin{abstract}
Software quality is very important for software, and software test is an important method of software quality assurance. This paper provided one scheme for software testing management based on TestCenter. This research applied TestCenter to manage the tests process. The software testing process focused on test requirements, test plans, test execution and bug trace, which can ensure test cases to fully coverage the test requirement in theory and trace bug in real time. Our practice proves that the scheme can shorten cost time of test project effectively.
\end{abstract}

Keywords-software testing management; TestCenter; bug track; test requirement; test plan

\section{INTRODUCTION}

Generally, in the case of a large-scale software development, more than the half of the total development effort is said to be spent on the testing phase. Therefore, the management of the fault-detection and fault-correction activities in the testing phase is very important to efficiently and economically develop a highly-reliable software product[1]. However, traditional testing work is managed by document. We often use word and excel to write test case without nay test management tool. Software testing procedure is time-consuming and inefficiency only by human method.

The main problem that exists in software testing management research is how to effectively manage the whole software test processes in a single environment.[2]

Software testing management can reduce economic cost, and can make the test procedure under control. Test management tool can manage the whole testing procedure from several segments which are test requirement, test plan, test execution, bug trace and test summary. Through software testing management tool, we can monitor the whole test processes, and judge test team progress objectively. And then we can enhance test efficiency of test team. We choose ShangHai Spasvo Testcenter as test management tool, and combine version control software SVN to present one test management scheme.

\section{RELATED WORKS}

Scholars at home and abroad have taken up large-scale research on test management tool application. There are some typical scheme shows as below.

Wang Wei analyzed the features and limitations of the existing domestic and international software test management tools. Combined with the test requirements of military software test in our country and the relevant specification, analyzes the application of software testing management tools in each link of the military software testing process. Use software testing management tools can overcome by artificial test management limits, strengthen the test work of the organization and management. To ensure the accuracy of test work, consistency at the same time, the effective control of test quality, coordination test resources better, improve the testing efficiency. The research content of this paper provides an important reference for military software testing[3].

Wang YunPeng etl introduced one testing management scheme based on Quality Center. Quality Center was a kind of testing management tool for the whole life cycle of software product based on $\mathrm{B} / \mathrm{S}$. The tool integrated parts of testing management into an application system, which was include demanding management, testing plan, testing execution, bug management and statistics analysis. It could manage the testing process object objectively, and ensure tests fully coverage requirements, and reduce the burden software testing worker [4].

Zhang Jie discussed how to integrate software testing with development in the existing production in small and medium enterprises in order to improve the quality. Some commonly used software test tools and test management tools are introduced. The 80: 20 problem that should be noticed in the software testing management is explained. The practice shows that using the entire software testing management is helpful in solving the test problems existing in the management. Some software testing tools were compared, which includes TestDirect, HP QualityCenter, Spasvo TestCenter, IBM Rational TestManager, TestLink, etc[5].

Li ChangXing et al. introduced the software automated testing technology and software automated testing tools. They associated with project test system, the importance of software testing in software quality assurance process is introduced. Then the basic concept of software test is introduced and the testing life cycle of project test system is analyzed. Based on the project test system, the usage of webbased test tool Test Director in requirements management, test plan, test execution and defects tracing of test manage process is analyzed. Thereby the usability of the testing system is proved in practice, Meanwhile, the core role of automation test tool in development and test process is introduced[6]. 
Fujiwara $\mathrm{T}$ et al present a software testing-management tool which can support the quality/reliability assessment and testing-progress control in the testing phase is developed. Five software reliability growth models(SRGM's), i.e. exponential SRGM, delayed S-shaped SRGM, testingdomain dependent SRGM, testing-domain dependent SRGM with skill-factor which are based on a nonhomogeneous Poisson process, and the Gompertz curve model which is a deterministic regression model, are built into the this tool. They implement this tool by using a $\mathrm{C}++$ language. Finally, application examples of this tool to the fault data observed in an actual development project are shown[7].

Considering some kinds of software-testing management problems, such as requirement traceability, test coverage and testing-resource control problem for how to spend the allocated amount of testing-resource per expected time. Safana A et al apply SpiraTeam to manage the tests on an embedded real time system of medium size called OBA. They report and demonstrate the capabilities of SpiraTeam to manage test activities and its significant contribution in test management[8].

\section{TESTCENTER INTRODUCTION}

TestCenter is an excellent test management tool, and its main function shows as below[9]:

- Manage test requirement: it can manage test requirement by item and organize them by test requirement tree. Every test requirement item is described by detailed information. Test requirement is linked to test case or bug, and linked to scene also. Under the scene we can design scene step and scene data.

- $\quad$ Manage test plan: it can support manage test plan. Every test plan includes test resource, test task, test cycle and so on. It can support to check test report by test plan. Test plan is organized by test version, test plan and test cycle three level.

- Manage test case: it can construct test cast tree with same structure of test requirement. It can support test case description, such as test target, test requirement belong to, test step, test data and information about automatic test. Test case generation method mainly includes manual scene method and model driven case method.
- Generate test set: it can provide a tool for project manager to execute test plan. Test case can't run alone, so one test set contains many test cases. Test set is a unit to start test execution. Test set must be linked to test cycle in test plan.

- Test execution: it can organize test case by test target to inform test set and execute test set. Test execution is usually started by project manager, and then it starts to bug trace.

- $\quad$ Bug trace: it can support define customer workflow of bug trace, and also support define customer bug status and customer user role.

- Test report generation: It can generate some reports, for example test cycle report and test summary report. It can generate customer report.

\section{TEST SCHEME RESEARCH}

We present one test management scheme based on TestCenter. First, we introduce software resource configuration, which mainly includes TestCenter and SVN. Then, we introduce execution process which is implemented by test requirement, test plan, test execution, bug trace and statistical analysis.

\section{A. Software Configuration for Test Team}

Test center is the Core tool of our scheme. TestCenter is used by project manager, test leader, development leader, test engineer and development engineer. Test center manage test requirement, test plan, test case and bug. Each role accesses corresponding resource according to each authority. In addition, except for tracing test process, we adopt SVN to memory test document. Test leader is responsible for test document, such as test plan, function test report and performance test report. Development leader is responsible for development document, such as requirement analysis document, summary design document, and detailed design document. Other role can only read the document but not allowed to write or update the document, which can assure document accuracy. Automatic test tool, for instance AutoRunner and PerformanceRunner, is used by test leader and test engineer. Fig.1 shows test management software configuration, we can extend configuration with more automatic test tool. 


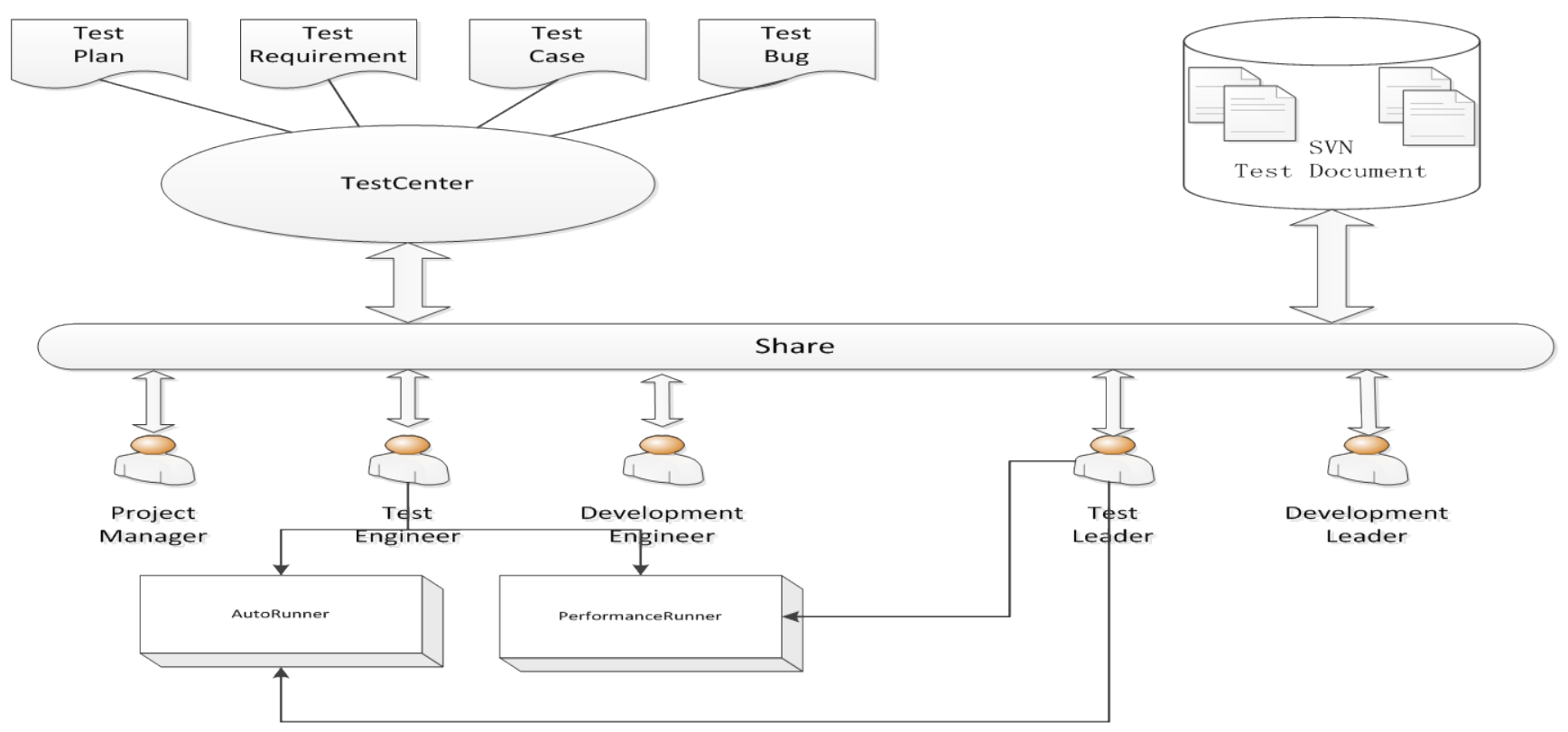

Figure 1. Test Team Software Configuration.

\section{B. Test Process Implement}

The test process will be implemented through test requirement stage, test plan stage, test execution stage, bug trace stage and statistical analysis stage. Fig.2 shows the main concept for each stage. We explain working content for the five stages.

1) Test requirement: Now we introduce the first sate. Test case management is the key problem for TestCenter. The tracing source of test case design is test requirement. So, when we design test case we must consider test requirement. We must make clear what function do you want to test. And we must make clear how the software run and what is the input and output. Test requirement mainly explain what will you test. TestCenter provides test requirement, test sub requirement and case scene to describe test requirement. In fact, test requirement is depend on software requirement analysis from development. As test team worker, we focus on understanding software requirement, and input them into TestCenter.

2) Test plan: In fact, the target of test plan is to manage test case. TestCenter manage many test cases through test version, test plan, test cycle and test set. Entering the next test execution stage, we can implement test execution by the several stages. Test version is linked to many test requirements. Test set is linked to many test cases. We can check whether test cases are full coverage of test requirement, and assure the test quality.

\section{Test Execution}

Test execution is relatively simple to understand. We can't start execute single test case in TestCenter. We can start test execution with test set. When test engineer finds bug, he will submit the bug into TestCenter, and then TestCenter will trace the bug in the whole lifecycle.

\section{Bug Trace}

TestCenter is allowed to define customer bug lifecycle. The customer bug process that we adopted shows as Fig.3 The whole lifecycle of bug includes new, confirm, allocate, reject, updating, update complete, reopen and close. A bug starts with begin status and ends with end status. Each test team can define customer bug lifecycle according to its own feature. Fig. 3 shows one kind of customer bug lifecycle. A bug starts with new status and ends with close status. The complete bug lifecycle contains some status, such as confirm, allocate, reject and so on. A bug is transmitted by different role in the team. 


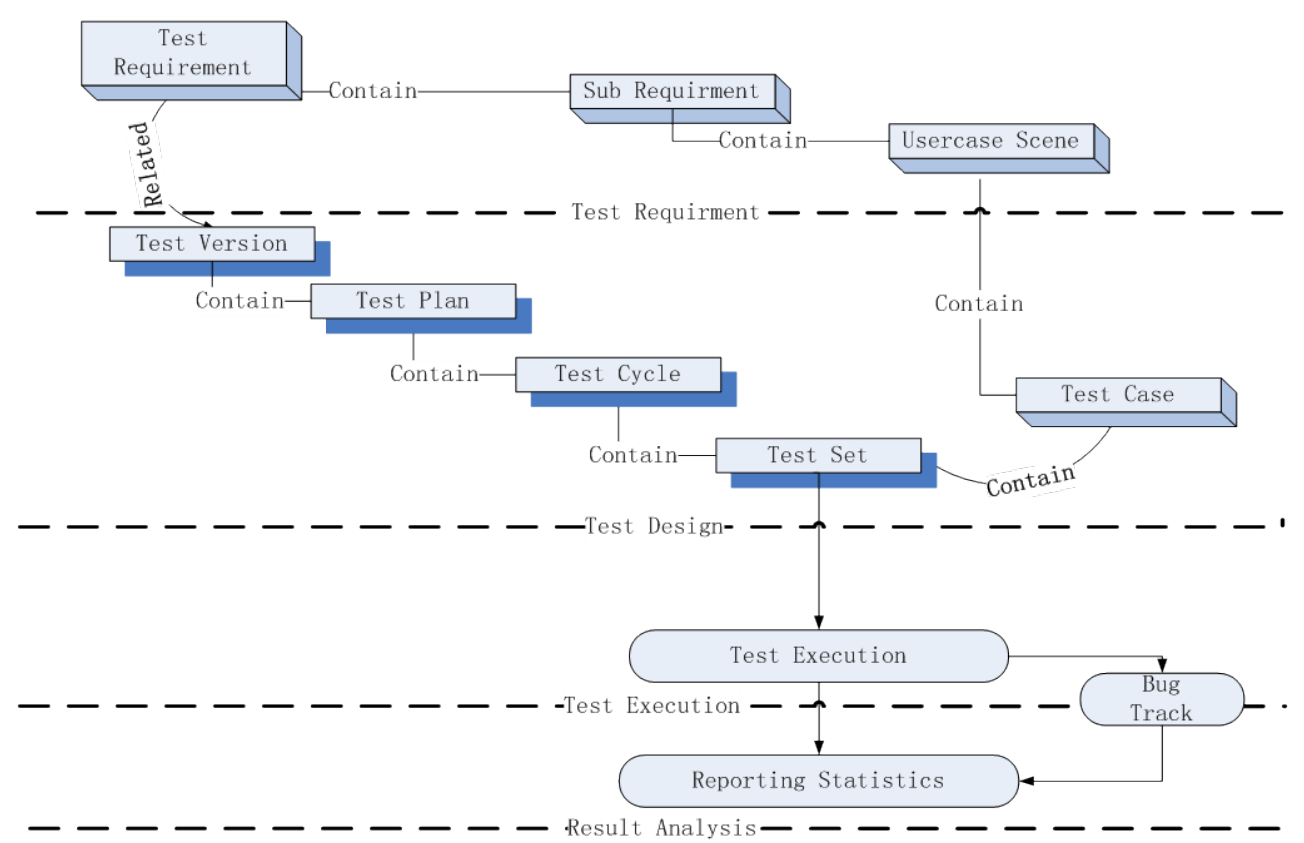

Figure 2. Test Procedure Based on TestCenter.

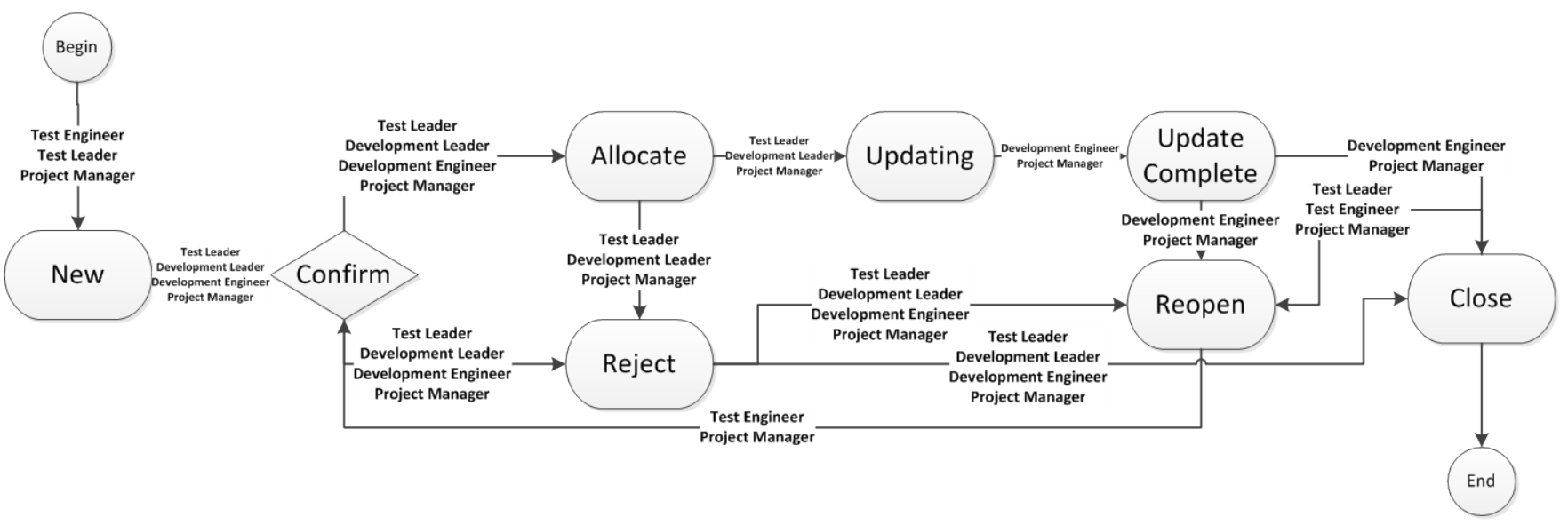

Figure 3. Customer Bug Lifecycle.

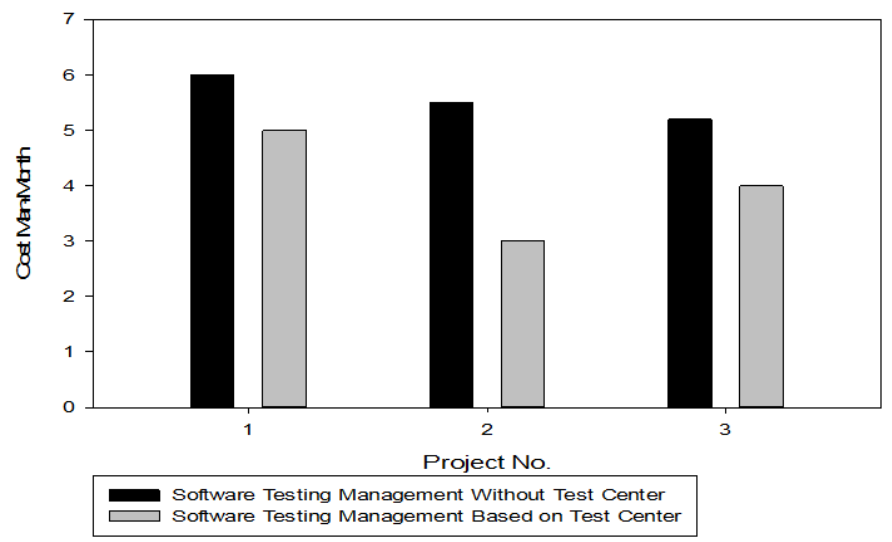

Figure 4. Comparison Man-Month Cost Between Using TestCenter and Not Using TestCenter. 


\section{E. Statistical Analysis}

TestCenter can provide abundant statistical report for test team to trace project process. It can provide cycle process report, cycle test report, bug view, bug statistical report, test summary report and so on. We can choose some typical report as data report for test document.

Above all, TestCenter is a high-integrated test management tool. If the core achievement of development team is source code, the core achievement of test team is test case and bug. No matter how complicated the test management tool is, they are constructed by the two core content-test case and bug.

The scheme we presented implemented three projects. Fig.4 shows the comparison between using TestCenter and not using TestCenter. Working efficiency is enhanced by $15 \% \sim 20 \%$. With the practicing TestCenter more skillful, test project cost time will be shortened further.

\section{CONCLUSION}

In order to make software tests process flexible and changeable, we recommend the use of TestCenter. TestCenter can coverage the whole software test process which includes test requirement, test plan, test execution, bug trace and result analysis. TestCenter helps us to assure coverage rate of test requirements, and enhance test team implement quality.

\section{ACKNOWLEDGMENTS}

This research was financially supported by the Wuhan polytechnic excellent course.

\section{REFERENCES}

[1] Fujiwara T, Yamada S, Sekine K, et al. A Software TestingManagement Tool(SAFEMAN)and Its Application[J]. Ieice Technical Report Reliability, 2000, 100:31-36.

[2] Safana A I, Ibrahim S. Implementing Software Test Management Using SpiraTeam Tool.[C]// International Conference on Software Engineering Advances, Icsea 2010, 22-27 August 2010, Nice, France. 2010:447-452.[M]

[3] WANG Wei, WANG Li, LiDa. The Usage of Software Testing Management tools in Military Software Test[J]. Computer Security, 2014(3):23-27.

[4] WANG YunPeng, HE XiaoHui, Testing management based on Quality Center[J].Railway Computer Application, 2014, 23(3):37-40.

[5] Zhang Jie. Discussion of software test tools analysis and test management [J]. Computer Era, 2014(6):50-52

[6] Li ChangXing, Shi Jing. Test Director in the test management application [J]. Electronic Test, 2013(3):76-79.

[7] Fujiwara T, Yamada S, Sekine K, et al. A Software TestingManagement Tool(SAFEMAN)and Its Application[J]. Ieice Technical Report Reliability, 2000, 100:31-36.

[8] Safana A I, Ibrahim S. Implementing Software Test Management Using SpiraTeam Tool.[C]// International Conference on Software Engineering Advances, Icsea 2010, 22-27 August 2010, Nice, France. 2010:447-452.

[9] http://www.spasvo.com 